\title{
Pervasive joint influence of epistasis and plasticity on mutational effects in Escherichia coli
}

\author{
Susanna K Remold ${ }^{1,2}$ \& Richard E Lenski ${ }^{1}$
}

The effects of mutations on phenotype and fitness may depend on the environment (phenotypic plasticity), other mutations (genetic epistasis) or both. Here we examine the fitness effects of 18 random insertion mutations in $E$. coli in two resource environments and five genetic backgrounds. We tested each mutation for plasticity and epistasis by comparing its fitness effects across these ecological and genetic contexts. Some mutations had no measurable effect in any of these contexts. None of the mutations had effects on phenotypic plasticity that were independent of genetic background. However, half the mutations had epistatic interactions such that their effects differed among genetic backgrounds, usually in an environment-dependent manner. Also, the pattern of mutational effects across backgrounds indicated that epistasis had been shaped primarily by unique events in the evolutionary history of a population rather than by repeatable events associated with shared environmental history.

How common are epistasis and plasticity? How do they arise during evolution? The evolutionary consequences of population subdivi$\operatorname{sion}^{1,2}$, the maintenance of $\operatorname{sex}^{3-5}$ and the role of environmental heterogeneity in speciation ${ }^{6}$ all depend on the answers to these questions. Most studies that have examined the effects of individual alleles in multiple environments or genetic backgrounds have focused on particular genes already known to have context-dependent function ${ }^{7-10}$.

Figure 1 Plasticity and epistasis of mutations illustrated and tested. (a-d) Column 1, types of interaction. The corresponding sources of variation from mixed linear models are: a, current environment; $\mathbf{b}$, genetic background; $\mathbf{c}$, interaction of environment and background; $\mathbf{d}$, no effect of environment, background, or their interaction. Column 2, illustrative reaction-norm patterns. Column 3, number of mutations showing each pattern at $P<0.05$. Column 4, fitness effects (means and $95 \%$ confidence intervals) of example mutations. Graphs show fitness effects of a single mutation in two environments ( $G$, glucose; $M$, maltose; bottom axis), with ancestor (dashed), glucose-evolved (black) and maltose-evolved (gray) backgrounds indicated by separate lines. The corresponding graphs for all 18 mutations are shown in Supplementary Note 1.

$\begin{gathered}\text { Type of } \\ \text { interaction }\end{gathered}$
$\begin{gathered}\text { Illustrative } \\ \text { case } \\ \text { only }\end{gathered}$

b

Epistasis only

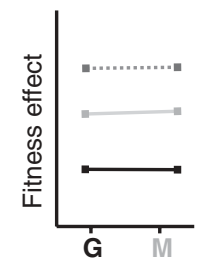

C

Both

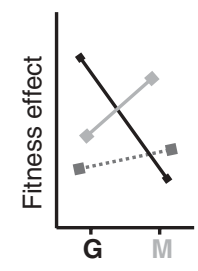

$6 / 18$

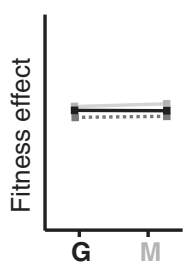

$9 / 18$

d

Neither
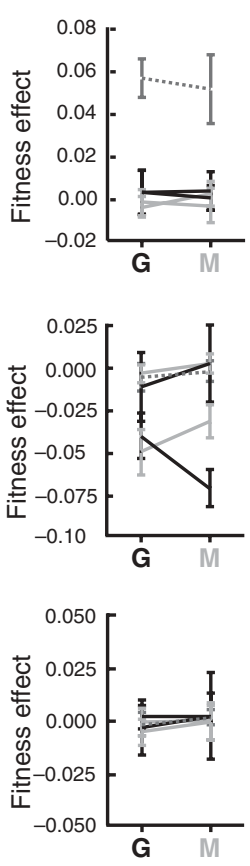

${ }^{1}$ Center for Microbial Ecology, Michigan State University, East Lansing, Michigan 48824, USA. ${ }^{2}$ Current address: Department of Ecology and Evolutionary Biology, Osborn Memorial Laboratories, 165 Prospect Street, Yale University, New Haven, Connecticut 06520, USA. Correspondence should be addressed to S.K.R.

(susanna.remold@yale.edu).

Published online 14 March 2004; doi:10.1038/ng1324 
Table 1 Fisher's tests of combined probability from mixed models of effects of 18 insertion mutations

\begin{tabular}{lcc}
\hline Source & d.f. & $\chi^{2}$ \\
\hline Fixed & & $27.84^{\mathrm{ns}}$ \\
$\begin{array}{l}\text { Current environment } \\
\text { Random }\end{array}$ & 36 & \\
Genetic background & 36 & $297.33^{* * *}$ \\
Genetic background $\times$ current environment & 36 & $119.01^{* * *}$ \\
Block & 36 & $39.75^{\mathrm{ns}}$ \\
\hline
\end{tabular}

Mixed models for individual mutations are provided in Supplementary Note 1. ns, $P>$ $0.1 ;{ }^{* *}, P<0.001$

These studies therefore cannot address how commonly epistasis and plasticity influence the effects of alleles. Other studies have examined epistatic interactions involving random mutations, but have focused on a single environment and hence do not address whether these mutations might also affect phenotypic plasticity ${ }^{11-14}$. Still other studies have demonstrated variability in the effects of mutations on phenotypic plasticity but did not test for epistatic effects ${ }^{15,16}$, although one indicated that the pattern of mutational effects on plasticity in response to stress also implied epistasis ${ }^{16}$.

We examined the prevalence and evolutionary origins of epistasis and plasticity by quantifying the fitness effects of mutated alleles at randomly chosen loci in multiple growth conditions and genomes. We used an E. coli system in which genomes with different ecological histories served as genetic backgrounds for testing the effects of mutations. These backgrounds were chosen to distinguish between two scenarios. According to one, the environment generates repeatable adaptation across independent populations, resulting in changes with

\section{Selection on new allele}

\section{Illustrative cases}

a

Repeatable



b

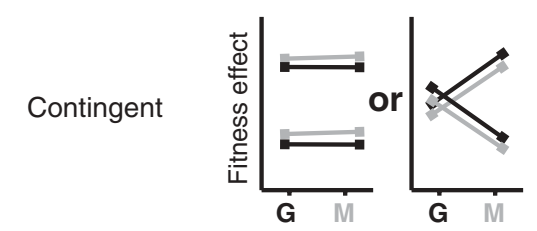

$3 / 18$
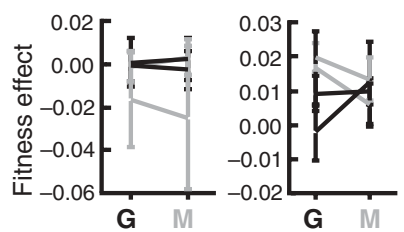

Glucose evolved

Maltose evolved

Figure 2 Epistasis reflecting repeatable versus contingent historical effects illustrated and tested. $(\mathbf{a}, \mathbf{b})$ Column 1 , types of selection. The corresponding sources of variation from mixed linear models are: $\mathbf{a}$, ecological history, or interaction of ecological history and current environment; $\mathbf{b}$, genetic background nested within ecological history, or interaction of background and environment nested within ecological history. Column 2, illustrative reaction-norms. Column 3, number of mutations showing each pattern at $P<0.05$. Total is $<18$ owing to mutations whose effects did not differ significantly among the backgrounds (excluding ancestor). Column 4 , fitness effects of example mutations, with graphs as in Figure 1. similar effects on genetic architecture. The effect of a particular mutant allele would then be more similar in genomes from populations that shared a common ecological history than in genomes that had adapted to different environments. Alternatively, independent adaptation by replicate populations to the same environment might produce divergent architectures, such that subsequent selection on new mutations would be historically contingent even for replicate populations that shared the same ecological history ${ }^{1,2,6}$.

We transduced 18 mutations into 5 genetic backgrounds each ${ }^{17}$. We selected the mutations from a set of mutants carrying mini-Tn10 transposons inserted randomly throughout the E. coli genome ${ }^{13,18}$. Transductions conserving each insertion's location were made into two genomes isolated from different populations that evolved for 1,000 generations on glucose, two genomes from different populations ancestral genome. The pairs of populations that shared ecological histories experienced parallel fitness gains in their own selective environments, and they showed a mix of parallel and divergent changes in traits, including their performance in different environments ${ }^{19-21}$. We carried out competitions to estimate the fitness effect of each insertion mutation in both the glucose and maltose environments and in all five genetic backgrounds, with fivefold replication, for eplications) each lasting 40 generations.

Possible effects of mutant alleles are shown as reaction norms ${ }^{22}$ in Figure 1. If the fitness effect of a mutation depends only on the current environment, and if it influences phenotypic plasticity consistently across genetic backgrounds, then all reaction-norm lines will share the same height at their midpoints and the same significant slope (Fig. 1a). Although earlier work showed extensive environment-dependent effects of insertion mutations in a single genetic backgound ${ }^{15}$, our analyses of these 18 mutations detected no fitness effects that were environment dependent but genetic background independent at either the $\alpha=0.05$ or $\alpha=0.1$ level (see Supplementary Note 1 online for individual analyses of each mutation and reaction norms depicting each mutations' fitness effects). This number is lower than the approximately one significant outcome expected based on chance alone. Fisher's test for combined probabilities among the 18 mutations also found no overall significant effect of current environment (Table 1). Thus, mutations with consistent effects on phenotypic plasticity across the genetic backgrounds are rare.

If a mutation's fitness effect depends on genetic background and its epistatic interactions are not environment dependent, then the reaction-norm lines will differ significantly in their heights but share a slope of zero (Fig. 1b). Three mutations (16\% of total) affected fitness differently among backgrounds but not among environments at $P<$ 0.05 . The combined-probabilities test also found highly significant genetic backgrounddependent fitness effects (Table 1). Thus, some of the introduced mutations interact epistatically with other mutations that differ among the five genetic backgrounds. 
If a mutation's effect depends on both environment and genetic background, then its reaction-norm lines will differ in slope (Fig. 1c). Six mutations (33\%) had significant joint plastic and epistatic effects at $\alpha=$ 0.05 and eight mutations (44\%) did so at $\alpha=0.1$. For example, some were neutral in glucose and deleterious in maltose in one background, but showed no environmental dependence in other backgrounds. The combined-probabilities test also found highly significant environmentby-background interactions (Table 1). Moreover, a Fisher's exact test of the numbers of mutations showing plastic and epistatic effects showed significant nonindependence of these two properties (two-tailed, $P=$ 0.001 ). That is, mutations for which environmental and genetic interactions jointly influence fitness were more common than expected from the prevalence of each interaction type alone.

Finally, if a mutation does not show environmental or genetic interactions, then its reaction-norm lines will share a common height and zero slope (Fig. 1d). Nine mutations (50\%) were not significantly affected by either the environment or the genetic background using an $\alpha$ level of 0.05 . Of these, seven did not differ significantly from neutrality. The other two had significant but small negative fitness effects. Thus, loci with consistent and large effects across environments and backgrounds are fairly rare, and descriptors such as 'deleterious' and 'beneficial' are usually context dependent.

To understand how ecological histories affected the emergence of epistasis and plasticity, we analyzed mutational effects in the four evolved backgrounds (excluding the ancestor). If the populations that independently evolved under the same conditions (glucose or maltose) experienced parallel changes in their genetic architectures, then reaction-norm lines for mutations in these backgrounds should be more similar than lines for backgrounds that evolved under different conditions (Fig. 2a). Further dependence of the effects of mutations on the current environment might be present or absent as shown by illustrative cases. However, none of the 18 mutations had effects that significantly depended on the particular ecological history of the recipient genetic background based on an $\alpha$ level of 0.05 , and only one did so at $\alpha=0.1$ (see Supplementary Note 1 for individual analyses). The combinedprobabilities test also found no significant effect of ecological history nor any significant interaction between ecological history and current environment (Table 2: fixed effects involving ecological history).

Alternatively, the fitness effect of each new mutation could be contingent on random historical events, such that even genetic backgrounds with shared ecological histories would differ in their interaction with new mutations. If so, the reaction-norm lines for mutations in backgrounds that shared ecological histories should differ in slope or midpoint height (Fig. 2b). Indeed, 3 of 18 mutations had significantly different fitness effects in genetic backgrounds that had evolved in the same environment at $P<0.05$, and 2 more showed marginally significant differences $(P<0.1)$. This number is about three times as high as expected by chance alone $(17 \%$ of the mutations rather than the expected $5 \%$ at $P=0.05$, and $28 \%$ rather than the expected $10 \%$ at $P=$ 0.1 ). The combined-probabilities test confirmed that genetic backgrounds with the same ecological history strongly influence both the overall and current environment-dependent effects of mutations (Table 2: random effects nested within ecological history).

In summary, our experiments show that many mutations affect fitness in ways that depend on the precise environmental and genetic context in which they are tested. In addition, those mutations that show epistasis (dependence on genetic background) are disproportionately likely also to show phenotypically plastic effects (environmental dependence). To the best of our knowledge, this association has not previously been demonstrated, although a recent study implied a conceptual connection between these two types of interaction ${ }^{16}$. The epistatic
Table 2 Fisher's tests of combined probability from reduced mixed models (excluding ancestor) of effects of 18 insertion mutations

\begin{tabular}{lcc}
\hline Source & d.f. & $\chi^{2}$ \\
\hline Fixed & & \\
Current environment & 36 & $22.80^{\mathrm{ns}}$ \\
Ecological history & 36 & $32.38^{\mathrm{ns}}$ \\
Ecological history $\times$ current environment & 36 & $22.09^{\mathrm{ns}}$ \\
Random & 36 & $142.03^{* * *}$ \\
Genetic background (ecological history) & & $73.72^{* * *}$ \\
Genetic background $\times$ current environment & 36 & $42.69^{\mathrm{ns}}$ \\
(ecological history) & 36 & \\
Block & &
\end{tabular}

Mixed models for individual mutations are provided in Supplementary Note 1. ns, $P>0.1$; $* * *, P<0.001$.

effect of genetic background arose quickly in evolutionary terms (within 1,000 generations since a common ancestor) and even when the backgrounds had diverged in the same selective environment. Thus, closely related populations can have quite different genetic architectures for fitness, and perhaps for traits whose phenotypes evolve in parallel ${ }^{1,2}$. These findings also show that models incorporating both plasticity and epistasis may provide greater power and precision for plant and animal breeding programs ${ }^{23}$.

\section{METHODS}

Mutations. Our experiments used 18 insertion mutations, 9 each carrying resistance to kanamycin $\left(\operatorname{Kan}^{\mathrm{R}}\right)$ and tetracycline $\left(T e t^{\mathrm{R}}\right)$. These mutations were randomly chosen from a larger set of mutations ${ }^{13}$ generated by mini-Tn 10 transposon mutagenesis ${ }^{18}$ in a clone of $E$. coli $\mathrm{B}$ isolated from a population that had evolved for 10,000 generations in a minimal medium supplemented with glucose $^{24}$.

Transductions. We used Plvir to transduce the 18 insertion mutations into recipient backgrounds following the standard protocol ${ }^{17}$, and selected recombinants on LB agar with supplemental antibiotic. P1 transduction replaces $\leq 96 \mathrm{~kb}$ of recipient DNA with donor DNA through homologous recombination, while generally conserving the transposon's genomic location. Southern blotting confirmed the shared transposon locations between donors and recombinants.

We constructed 90 recombinants in all. We transduced the nine $\mathrm{Kan}^{\mathrm{R}}$ mutations into the ancestor of all the evolved recipients ${ }^{19,20}$, two clones isolated from independent populations evolved for 1,000 generations on glucose ${ }^{19}$ and two clones from populations evolved for 1,000 generations on maltose $\mathrm{e}^{20}$. We discarded one $\mathrm{Kan}^{\mathrm{R}}$ recombinant owing to its inadvertent acquisition of P1 resistance. Similarly, we transduced the nine $\mathrm{Tet}^{\mathrm{R}}$ mutations into the ancestor and into two glucose-evolved and two maltose-evolved clones from different populations than those receiving $\mathrm{Kan}^{\mathrm{R}}$ mutations. These two independent sets of evolved recipients were used to sample a broader range of responses to prior selection.

The fitness of a recombinant could, in principle, include unintended effects of markers and co-transduced alleles. Earlier experiments with these minitransposons found no effects of antibiotic markers ${ }^{15}$, and we did not detect Ara marker effects (as discussed later). Co-transduction of any mutations other than the transposon insertion is very unlikely because donor and recipient genotypes were always $\leq 11,000$ generations apart and thus differ at rather few loci ${ }^{25,26}$. Co-transduced alleles, if any, tightly linked to the transposon would be present in all five recombinants, and the effects thereof would reflect true interactions. A tightly linked allele could also have produced a false-positive effect of ecological history, but we did not detect such an effect (Table 2) and hence this potential complication is not relevant. To examine the potential effects of weakly linked and thus intermittently cotransduced alleles, we used P1 transduction to make 3-5 replicate transductions of 4 mutation-genetic background combinations, and carried out 3-5 replicate fitness assays in glucose and maltose for each combination as described below. We detected no significant overall or environment-dependent variability among replicate transductants and 
only one case of marginally significant variability among the four trial combinations. Fisher's test for combined probabilities also detected no significant overall $(P=0.99)$ or environment-dependent $(P=0.28)$ effects of replicate transductions among the trial combinations. Thus, significant variability in the effects of mutations resulting from weakly linked alleles is unlikely.

Fitness assays. We measured the fitness effect of each mutation in ten contexts: five genetic backgrounds and two environments. Each recombinant competed against a competitor identical to its progenitor (same background), except that these competitors can use arabinose $\left(\mathrm{Ara}^{+}\right)$whereas the progenitors and recombinants cannot $\left(\mathrm{Ara}^{-}\right)$. On tetrazolium-arabinose (TA) indicator agar, $\mathrm{Ara}^{-}$and $\mathrm{Ara}^{+}$cells form red and white colonies, respectively, enabling us to quantify changing densities in competitions ${ }^{19,27}$. Control competitions $\left(\mathrm{Ara}^{+}\right.$competitor versus isogenic $\mathrm{Ara}^{-}$progenitor) run concurrently with the experimental competitions showed no discernible effect of the Ara marker in any environment.

The two environments used in the fitness assays were identical to those used to derive the evolved progenitor genotypes ${ }^{19,20}$, except that the bacteria grew in unshaken tubes (rather than in shaken flasks). Competitors were grown separately for one serial-transfer cycle ( $\sim 6.6$ generations) to allow acclimation to the assay environment ${ }^{19}$. They were mixed at a 1:1 volumetric ratio, diluted 100fold into fresh medium and allowed to compete for six cycles ( $\sim 40$ generations $)^{12,13,15}$. At the initial and final time-points, the mixtures were plated on TA agar to ascertain their densities. We calculated each competitor's malthusian parameter as $m=\ln \left(N_{6} \times 100^{6} / N_{0}\right)$, where $N_{0}$ and $N_{6}$ are initial and final densities and the factor of $100^{6}$ reflects six 100 -fold dilutions. This parameter integrates growth and survival over all phases of competition in the serial-transfer regime. The relative fitness of each genotype is the dimensionless ratio of its malthusian parameter to that of its competitor ${ }^{19}$; the fitness effect (selection coefficient) is the relative fitness minus 1 .

Five replicate assays in randomized incomplete blocks were run for each of the two independent experiments (one involving the $\mathrm{Kan}^{\mathrm{R}}$ mutations, the other involving the $T e t^{\mathrm{R}}$ mutations). Each block consisted of one replicate competition of each of the nine mutations in the experiment in all genetic backgrounds and in both environments. This design resulted in 90 competitions per block. Incompleteness was generated by the replacement of three mutants that were found to have acquired P1 resistance (a fourth was not replaced and all mutants containing that insertion were dropped from the study); fitness assays of the replacements were always carried out with a complete replicate of all other backgrounds containing that mutation in both environments (five genotypes, ten competitions). Thus, with respect to the analyses of each individual mutation, all blocks are in fact randomized and complete (Supplementary Note 1).

Statistical analyses. We ran two mixed linear models controlling for block effects for each mutation ${ }^{28}$. The first included current environment, genetic background and their interaction. The second, run on reduced data (excluding the ancestor background), included current environment, ecological history, genetic background nested within ecological history, and interactions of the last two factors with current environment. We tested random factors using likelihood-ratio tests, which compare the restricted likelihood of nested models with and without the variance component of interest ${ }^{28}$. This type of test is asymptotically based and needs to be adjusted when the null hypothesis is on the boundary of the parameter space. Although approximate, this test has the advantage that it can be used for all of the hypotheses considered here, including those that test for heterogeneous variances (R. Wolfinger, personal communication). We used Fisher's method to test the significance of combined probabilities from the multiple tests of different mutations ${ }^{29}$. This method allows the testing of interactions of interest across all mutations from the two independent experiments involving different genetic markers and experimental backgrounds. We ran additional mixed linear models for each of the two independent experiments $\left(\mathrm{Kan}^{\mathrm{R}}\right.$ and $\mathrm{Te} t^{\mathrm{R}}$ mutations) in which the data sets were reduced to be fully balanced; these models corroborate the conclusions from other statistical analyses (see Supplementary Note 2).

Note: Supplementary information is available on the Nature Genetics website.

\section{ACKNOWLEDGMENTS}

We thank S. Elena and M. Travisano for sharing strains; N. Hajela for technical support; C. Borland, T. Cooper, H. Eisthen, B. Lundrigan, P. Moore and M. Travisano for valuable comments; and R. Wolfinger and C. Duarte for statistical advice. This work was supported by a fellowship from the US National Institutes of Health to S.K.R. and a grant from the National Science Foundation to R.E.L.

\section{COMPETING INTERESTS STATEMENT}

The authors declare that they have no competing financial interests.

Received 11 September 2003; accepted 9 February 2004

Published online at http://www.nature.com/naturegenetics

1. Wade, M.J. Epistasis as a genetic constraint within populations and an accelerant of adaptive divergence among them. in Epistasis and the Evolutionary Process (eds. Wolf, J.B., Brodie, E.D.I. \& Wade, M.J.) 213-231 (Oxford Univ. Press, Oxford, UK, 2000).

2. Wade, M.J. \& Goodnight, C.J. The theories of Fisher and Wright in the context of metapopulations: when nature does many small experiments. Evolution 52, 1537-1553 (1998)

3. Otto, S.P. \& Feldman, M.W. Deleterious mutations, variable epistatic interactions, and the evolution of recombination. Theor. Pop. Biol. 51, 134-147 (1997).

4. Hansen, T.F. \& Wagner, G.P. Epistasis and the mutation load: a measurement theoretical approach. Genetics 158, 477-485 (2001).

5. Kondrashov, F.A. \& Kondrashov, A.S. Multidimensional epistasis and the disadvantage of sex. Proc. Natl. Acad. Sci. USA 98, 12089-12092 (2001).

6. Whitlock, M.C., Phillips, P.C., Moore, F.B.-G. \& Tonsor, S.J. Multiple fitness peaks and epistasis. Annu. Rev. Ecol. Syst. 26, 601-629 (1995).

7. Dean, A.M. A molecular investigation of genotype by environment interactions. Genetics 139, 19-33 (1995).

8. Fedorowicz, G.M., Fry, J.D., Anholt, R.R. \& Mackay, T.F.C. Epistatic interactions between smell-impaired loci in Drosophila melanogaster. Genetics 148, 1885-1891 (1998).

9. Dykhuizen, D.E. \& Hartl, D.L. Functional effects of PGI allozymes in Escherichia coli. Genetics 105, 1-18 (1983).

10. Lenski, R.E. et al. Epistatic effects of promoter and repressor functions of the $\operatorname{Tn} 10$ tetracycline-resistance operon on the fitness of Escherichia coli. Mol. Ecol. 3, 127-135 (1994)

11. De Visser, J.A.G.M., Hoekstra, R. F. \& van den Ende, H. Test of interaction between genetic markers that affect fitness in Aspergillus niger. Evolution 51, 1499-1505 (1996).

12. Elena, S.F. \& Lenski, R.E. Test of synergistic interactions among deleterious mutations in bacteria. Nature 390, 395-398 (1997).

13. Elena, S.F., Ekunwe, L., Hajela, N., Oden, S.A. \& Lenski, R.E. Distribution of fitness effects caused by random insertion mutations in Escherichia coli. Genetica 102/103, 349-358 (1998)

14. Elena, S.F. \& Lenski, R.E. Epistasis between new mutations and genetic background, and a test of genetic canalization. Evolution 55, 1746-1752 (2001).

15. Remold, S.K. \& Lenski, R.E. Contribution of individual random mutations to genotype-by-environment interactions in Escherichia coli. Proc. Natl. Acad. Sci. USA 98, 11388-11393 (2001)

16. Kishony, R. \& Leibler, S. Environmental stresses can alleviate the average deleterious effect of mutations. J. Biol. 2, 14 (2003).

17. Miller, J.H. A Short Course in Bacterial Genetics (Cold Spring Harbor Laboratory Press, Plainview, New York, USA, 1992).

18. Kleckner, N., Bender, J. \& Gottesman, S. Uses of transposons with emphasis on Tn10. Meth. Enzymol. 204, 139-180 (1991).

19. Lenski, R.E., Rose, M.R., Simpson, S.C. \& Tadler, S.C. Long-term experimental evolution in Escherichia coli. I. Adaptation and divergence during 2,000 generations. Am. Nat. 138, 1315-1341 (1991).

20. Travisano, M. \& Lenski, R.E. Long-term experimental evolution in Escherichia coli. IV. Targets of selection and the specificity of adaptation. Genetics 143, 15-26 (1996).

21. Travisano, M. Long-term experimental evolution in Escherichia coli. VI. Environmental constraints on adaptation and divergence. Genetics 146, 471-479 (1997).

22. Schmalhausen, I.I. Factors of Evolution: The Theory of Stabilizing Selection (Blakiston, Philadelphia, USA, 1949).

23. Podlich, D.W. \& Cooper, M. Modelling plant breeding programs as search strategies on a complex response surface. in Simulated Evolution and Learning (eds. McKay, B., Yao, X., Newton, C.S., Kim, J.-H. \& Furuhashi, T.) 171-178 (Springer, Canberra, Australia, 1998).

24. Lenski, R.E. \& Travisano, M. Dynamics of adaptation and diversification: a 10,000 generation experiment with bacterial populations. Proc. Natl. Acad. Sci. USA 91, 6808-6814 (1994).

25. Lenski, R.E., Winkworth, C.L. \& Riley, M.A. Rates of DNA sequence evolution in experimental populations of Escherichia coli during 20,000 generations. J. Mol. Evol. 56, 498-508 (2003)

26. Lenski, R.E. Phenotypic and genomic evolution during a 20,000-generation experiment with the bacterium, Escherichia coli. Plant Breed. Rev. 24, 225-265 (2004).

27. Levin, B.R., Stewart, F.M. \& Chao, L. Resource-limited growth, competition and predation: a model and experimental studies with bacteria and bacteriophage. Am. Nat. 111, 3-24 (1977).

28. Littell, R.C., Milliken, G.A., Stroup, W.W. \& Wolfinger, R.D. SAS System for Mixed Models (SAS Institute, Cary, North Carolina, USA, 1996).

29. Sokal, R.R. \& Rohlf, F.J. Biometry (Freeman, New York, USA, 1995). 